\title{
Electrochemical Lithiation Cycles of Gold Anodes Observed by In- Situ High-Energy X-ray Diffraction
}

\author{
Philipp Bach $^{1,2,31}$, Irais Valencia-Jaime ${ }^{4,51}$, Uta Rütt ${ }^{6}$, Olof Gutowski ${ }^{6}$, \\ Aldo H. Romero ${ }^{5}$, Frank U. Renner*1,2,7 \\ ${ }^{1}$ Institute for Materials Research (IMO), Hasselt University, Wetenschapspark 1, 3590 Diepenbeek, Belgium \\ ${ }^{2}$ Department of Interface Chemistry and Surface Engineering, Max-Planck Institut für Eisenforschung, Max-Planck- \\ Straße 1, 40237 Düsseldorf, Germany \\ ${ }^{3}$ Center for Electrochemical Sciences — CES, Ruhr-Universität Bochum, Universitätsstraße 150, 44780 Bochum, \\ Germany \\ ${ }^{4}$ Centro de Investigacion y Estudios Avanzados del IPN, MX-76230, Querétaro, México \\ ${ }^{5}$ Department of Physics and Astronomy, West Virginia University, Morgantown, WV-26506-6315, USA \\ ${ }^{6}$ German Electron Synchrotron (DESY), Notkestraße 85, 22607 Hamburg, Germany \\ ${ }^{7}$ IMEC vcs IMOMEC, Wetenschapspark 1, 3590 Diepenbeek, Belgium \\ ${ }^{*}{ }^{1}$ These authors contributed equally; \\ Corresponding author, Email address: frank.renner@uhasselt.be (F.U.R.)
}

\begin{abstract}
Significant developments of Li-ion batteries will be necessary to cope with the growing demands in electromobility or home storage of (sustainable) electrical energy. A detailed knowledge on the microscopic processes during battery cycling will be increasingly crucial for improvements. Involved phase changes at ambient temperature often involve metastable intermediate states, making both experimental observation and theoretical prediction of process pathways difficult. Here we describe an in-situ high energy X-ray diffraction study following the initial alloying and dealloying of Li with an Au thin film model anode using ionic liquid electrolyte. Six different crystalline alloy phases were observed to be involved in the cyclic phase transitions. Apart from the here highest lithiated phase, $\mathrm{Li}_{3} \mathrm{Au}$, none of the observed phases could be related to known, thermodynamically stable Li-Au phases. Structural search calculations following the minima hopping method (MHM) allowed the assignment of these phases to distinct metastable Au-Li alloy unit cells.
\end{abstract}




\section{Introduction}

Portable consumer electronics has taken a tremendously large part in today's modern work habits and lifestyle. Yet, current lithium ion battery (LIB) technology needs significant improvements to fulfill the requirements for future electromobility or home storage applications ${ }^{1-6}$. Recent LIB developments include for example Si-based alloy anodes, which offer a much higher volumetric and gravimetric capacity. ${ }^{7-11}$ Further examples are high voltage cathodes or general trends towards nanostructured components. ${ }^{9-12}$ In addition to finding cheaper production processes and materials, rechargeable (secondary) batteries also need to become more robust with longer lifetime in use. Fundamental insights into the acting atomic-scale processes and into the structural evolution within the active materials during the charging cycles, i.e. during lithiation and delithiation, is therefore required more than ever. Alloying reactions play a role not only in battery processes but also in many other electrochemical applications such as electrodeposition ${ }^{13}$ or nanotechnology ${ }^{14,15}$ where stepwise or cyclic alloying and dealloying may lead to (hierarchical) nanoporous metals. ${ }^{16-19}$ Phase transitions of solids are usually studied at elevated temperatures to reach quickly the thermodynamic stable structural states by fast diffusion. The phase changes of the active LIB electrode materials involved in the electrochemical charge transfer occur typically at ambient temperatures. Metastable, often amorphous ${ }^{20-24}$, intermediate states thus make both, experimental observation as well as theoretical prediction of the charging and discharging pathways, difficult.

Due to its inert nature and its large scattering factor Au has been used as a model material in electrochemical research, for example recently in in-situ TEM studies ${ }^{25-27}$. Furthermore Au was employed in LIB anodes as seed layers used to grow Si nanowires ${ }^{8,23}$ or as coatings or support ${ }^{28-31}$. For gold model electrodes or gold contained in a LIB anode several studies have pointed to unknown intermediate and metastable crystalline Li-Au phases ${ }^{24,30,32}$. X-ray diffraction is a classical non-destructive method to characterize crystalline phases, and synchrotron X-ray light has more recently facilitated the analysis of surfaces and thin films as well as insitu or in-operando characterization. Using in particular high-energy X-ray sources a large reciprocal space can be observed enabling precise measurement of structural changes on sub-second time scales. The benefits of high-energy X-rays have been demonstrated in-operando for detailed studies from catalytic surface reactions $^{33}$ to molecular interface structures ${ }^{34}$.

Single-crystal or thin-film model systems often allow detailed structural investigation of phase transformations of active material in electrodes avoiding other components such as binders or conductive additives. For electrolytes ionic liquids have been proposed as a promising alternative for $\mathrm{LIB}^{35-37}$. One important technical advantage is their low vapor pressure resulting in low inflammability. For basic research they offer the possibility to work at ultra-high vacuum (UHV) contributing to well-defined experimental conditions. 
Here we describe an in-situ high-energy X-ray diffraction (HEXRD) study following the initial alloying of Li (charging) and dealloying (dis-charging) with a Au thin film using ionic liquid electrolyte. We compare the experimental observations with a theoretical screening of possible, metastable phases. Addressing the model material $\mathrm{Li}-\mathrm{Au}$, we record a clear sequence of different crystalline phases including the known cubic $\mathrm{Li}_{3} \mathrm{Au}$ phase and several so far unknown metastable intermediates. With the presence of a sequence of crystalline phases in-situ high-energy X-ray diffraction is highlighted as a powerful tool to follow the structural evolution during complex reactions. Next to representing important battery reactions, lithiation and delithiation of $\mathrm{Au}$ was also employed to produce nanoporous $\mathrm{Au}$ films ${ }^{15}$ which are increasingly studied for various applications.

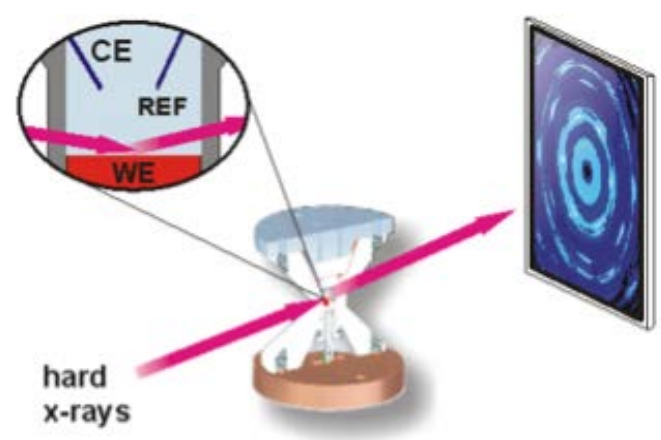

Figure 1: Setup for in-situ high-energy XRD experiments. The X-ray beam penetrates the PTFE-based electrochemical cell and a large part of the reciprocal space of the sample is imaged onto a $2 \mathrm{D}$ detector. Diffraction at different lattice planes occurs simultaneously and is thus visible in multiple Bragg peaks allowing the determination of the structural phases.

\section{Experimental}

The sample used for the presented electrochemical measurements was a $100 \mathrm{~nm}$ thin Au-film on a $\mathrm{Cu}(100)$ single-crystal substrate (MATECK GmbH, Jülich, Germany). The $\mathrm{Cu}(100)$ single-crystal substrate was oriented to better than $0.1^{\circ}$ and polished down to a RMS roughness of $0.03 \mu \mathrm{m}$. The surface was prepared in ultra-high vacuum (UHV) by sputtering/annealing. The Au film was grown by radio-frequency (RF) magnetron sputter deposition in an Ar atmosphere at room temperature at a deposition rate of $1 \AA /$ s. The Au sputter deposition was controlled by an Inficon IC6 deposition controller (INFICON Holding AG, Switzerland).

The in-situ High-Energy XRD (HEXRD) measurements were performed at beamline P07 at the storage ring PETRA III in Hamburg, Germany. The photon energy of the synchrotron radiation applied for the presented 
HEXRD experiments was $80 \mathrm{keV}$ corresponding to a wavelength of $\lambda=15.5 \mathrm{pm}$. The experiments were carried out in transmission geometry illuminating the film surface at grazing incidence (incident angle: $0.02^{\circ}$ ) with beam dimensions of 2-3 $\mu \mathrm{m}$ in height and 30-40 $\mu \mathrm{m}$ in width. The distance of the sample to the detector was 1396 mm. Debye-Scherrer diffraction rings were recorded using a PERKIN ELMER XRD 1621 Flat Panel 2D-detector. With an area of $41 \mathrm{~cm} \mathrm{x} 41 \mathrm{~cm}$ this detector is composed of $2048 \times 2048$ pixels, each of $200 \mu \mathrm{m} \times 200 \mu \mathrm{m}$ in size. The saturation is reached at 65 kcounts per pixel. A short readout time of $66 \mathrm{~ms}$ allows for a high time resolution. Here, sufficient counts were obtained within 1 sec of exposure time. Figure 1 shows a sketch of the diffraction setup.

Electrochemical treatment was carried out in a custom made electrochemical in-situ cell ${ }^{13,38}$ and a new Teflon cell designed especially for in-situ HEXRD experiments ${ }^{39}$. Li foils (KISCO Ltd., Tokyo, Japan) served as counter and reference electrodes. The electrochemical cell was assembled inside a glovebox (SYLATECH GmbH, Walzbachtal. Germany) under Argon atmossphere with $\mathrm{O}_{2}$ and $\mathrm{H}_{2} \mathrm{O}$ contents below $1 \mathrm{ppm}$. The electrolyte used was a $0.3 \mathrm{M}$ solution of the Li-salt LiTFSI (MERCK KGaA) in the ionic liquid $\mathrm{Pyr}_{14}$ TFSI (1-butyl-1-methylpyrrolidinium-bis(trifluoromethane-sulfonyl)-imide) ${ }^{37}$

(SOLVIONICS, Toulouse, France). Li foil was used for the counter and reference electrodes. After assembly the electrochemical cell was kept under Ar atmosphere and brought to the experimental station. During the experiment the cell was continuously flushed with Argon.

The electrochemical lithiation-de-lithiation cycles were performed by means of an Ivium CompactStat potentiostat (IVIUM Technologies B.V., The Netherlands). Galvanostatic cycling was performed at a current of $16.6 \mu \mathrm{A}(1,66 \mathrm{C})$ between potential limits of $1 \mathrm{~V}$ and $5 \mathrm{mV}$. The initial 2D diffraction patterns were radially integrated for further evaluation into 1D data using the QXRD software ${ }^{40}$.

The minima hopping method (MHM) $)^{41,42}$ which employs an efficient dynamical algorithm to explore the global minimum on the potential enthalpy surface was used to assist a structure determination. The evaluation of energy and forces required for MHM were obtained from DFT as implemented in the Vienna Ab initio Simulation Package (VASP). ${ }^{43,44}$ The efficiency of the escape step is improved by choosing the initial velocities of molecular dynamics trajectories approximately along soft mode directions. ${ }^{41}$ The projected augmented wave (PAW) method was used to describe valence and core electrons. ${ }^{45}$ To approximate the DFT exchange-correlation functional, we used the Perdew-Burke-Ernzerhof $(\mathrm{PBE})^{46}$ generalized gradient approximation. We used a plane wave cutoff of $650 \mathrm{eV}$, and the k-mesh used to sample the Brillouin zone was converged to guarantee a numerical accuracy of the total energy to less than 2 meV/atom. 


\section{Results and Discussions}

After assembling the in-situ electrochemical cell in the Ar-filled glovebox, the cell was mounted on the Xray diffractometer. The in-situ XRD measurements have been obtained employing synchrotron light of $80 \mathrm{keV}$ (15.5 pm) using different electrochemical cells. ${ }^{13,39}$ We followed the diffraction signal on a 2D detector as a function of galvanostatic lithiation and de-lithiation currents applied to Au thin-film model anodes of about 100nm in thickness. The respective potentials during such a measurement are shown in Figure 2a, where the letters correspond to the patterns shown in Figure 2b-g, respectively. From the diffraction patterns of the pristine sample (Fig. 2b) a pronounced texture of the sputtered Au film on the $\mathrm{Cu}(001)$ single-crystal substrate is obvious. The 2D (plane) diffraction pattern shows only distinct diffraction peaks (Bragg peaks) apart from some diffraction rings of low homogeneously-distributed intensity which originate from the Teflon material of the in-situ cell. ${ }^{39}$ The intense $\mathrm{Cu}(002)$ reflection is located on the vertical 00L-axis reflecting the (001) orientation of the $\mathrm{Cu}$ single-crystal. In the chosen azimuthal position (sample rotation around the surface normal) other visible Cu diffraction peaks of high intensity are the (131) and (133) peak (not marked here). In consequence, the crystal is oriented with the (3-10) direction pointing to the front. The presence of a few additional low-intensity $\mathrm{Cu}$ peaks points to a small number of distorted crystal regions, possibly at the edges of the sample or related to mounting in the cell.

The sputtered Au film shows (111)-orientation parallel to the $\mathrm{Cu}(001)$ substrate surface as it is apparent in Fig.2b from the intense $\mathrm{Au}(111)$ peak being located on the vertical, also called specular, 00L-axis along the same direction as the $\mathrm{Cu}(002)$ reflection. The presence and location of off-specular diffraction peaks corresponding to $\mathrm{Au}$ directly confirms the presence of rotational domains instead of a single continuous, epitaxial $\mathrm{Au}$ film. White arrows indicate the off-specular Au diffraction signal. Without focusing here in detail on the epitaxial relations, the shown orientation represents the strongest local maximum in intensity with $\mathrm{Cu}(3-10) \| \mathrm{Au}(1-10)$ and $\mathrm{Au}(-110)$. The two domains of the Au film are expected based on the symmetry of the substrate-film system. For thicker Au films also stacking faults may occur during growth of the film facilitated by the low stacking fault energy in bulk Au.

Further presented states of the electrochemical charging and de-charging cycle are a partially charged state (Fig.2c), the fully lithiated state after electrochemical alloying down to $5 \mathrm{mV}$ (Fig.2d), a partially de-lithiated state (Fig2e), and the final state after dealloying up to $1 \mathrm{~V}$ (Fig.2f). In the diffraction pattern of the fully lithiated state (Fig.2d) the Cu Bragg peaks from the substrate are still present, but the Au peaks have vanished. Instead new diffraction rings and a set of new distinct Bragg peaks are present, indicating the formation of Li-Au alloy phases. In the final state (Fig.2f) the $\mathrm{Cu}$ peaks are still present, but the distinct peaks and to a large extent also the diffraction rings originating from Li-Au alloy phases have disappeared. However, no visible peaks or rings of crystalline Au have recovered in the final state. Obviously pure Au 

giving rise to a Li-poor amorphous Au alloy phase remaining after de-lithiation.
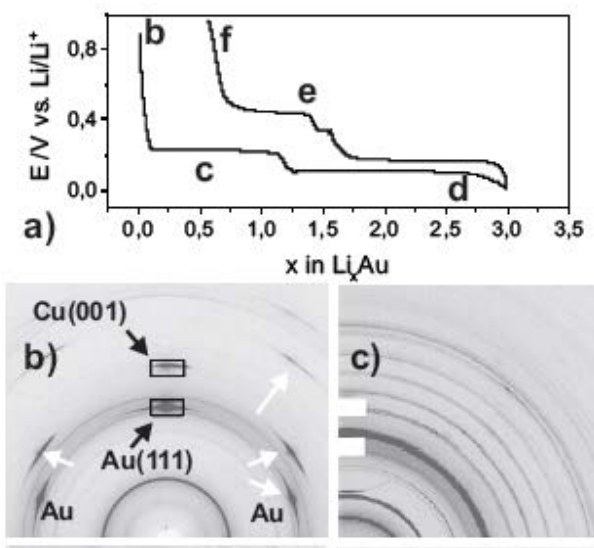

c)
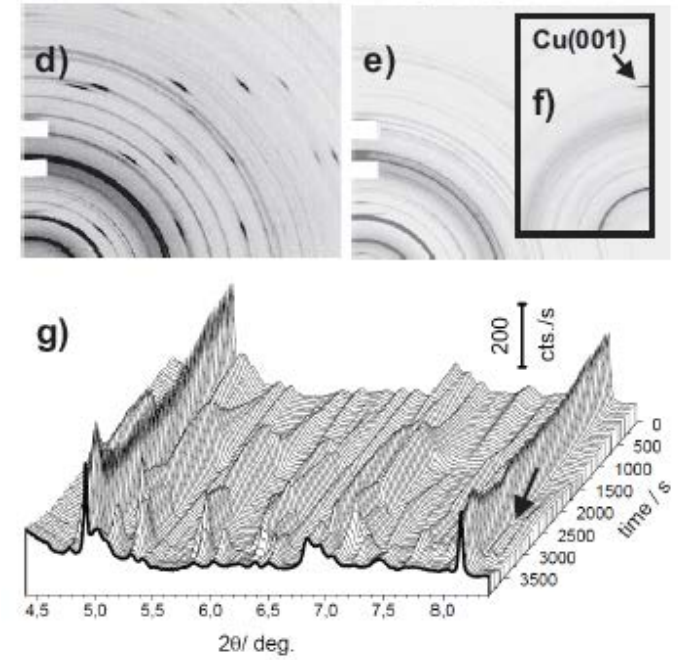

Figure 2: Selection of diffraction patterns. a) Typical galvanostatic lithiation cycle of a $100 \mathrm{~nm}$ Au thin film on $\mathrm{Cu}$ at $1.66 \mathrm{C}$ with reference to the displayed diffraction patterns below. b) Before the experiment the (111)-textured $\mathrm{Au}$ thin film is visible on the $\mathrm{Cu}(001)$ substrate (white arrows indicate off-specular $\mathrm{Au}$ diffraction signal). c) During lithiation branch. d) At the end of lithiation branch. e) During de-lithiation. f) At end of a full cycle no crystalline Au is recovered. g) Evolution of integrated diffraction patterns along one cycle.

For our in-situ XRD measurements we chose a time resolution of $6 \mathrm{~s}$. Diffractograms can be obtained from the individual diffraction patterns by radial integration. Arranging these in chronological order to a waterfall plot (3D intensity landscape) reveals similarities in the intensity-time behavior of different groups of the individual Bragg peaks. The intensity landscape represented in Fig. 2g appears complex and indicates that 
several different phases are involved in the lithiation-delithiation process of the Au thin film. The arrow at about 2700s shows the point where the current was reversed from lithiation to de-lithiation.

To separate and finally distinguish the involved different Li-Au alloy phases the evolution of intensity for all different Bragg peaks was compared. Bragg peaks following an identical trend in time were considered to belong to one structure. With this approach six clearly different groups of peaks were identified. Figure 3a represents the respective intensity trends for the first cycle and Figure $3 b$ for the third cycle. The cycle behavior is nearly identical starting from the second cycle. The full set of the different trends of the respective groups of Bragg positions is shown in Figure 4a. In this way we inferred the presence of different structural phases. The phases L1 and L2 are occurring during lithiation. L2 is the known, thermodynamically stable phase $\mathrm{Li}_{3} \mathrm{Au}$ with its composition in line with earlier electrochemical measurements. ${ }^{47}$ These phases appearing during lithiation as well as the phases DL1, DL 2, DL3, and DL4 appearing during de-lithiation were labeled along the specific sequence they reach their maximum in intensity during a cycle. In Fig. 3 the vertical line marks the point where the galvanostatic current was reversed to de-lithiation after the potential reached a set lower limit of $5 \mathrm{mV}$. At this point the intensity of phase L2 shows its maximum characterizing phase L2 as the highest lithiated Li-Au alloy phase. Apart from the intensity of phase L2 the evolutions of intensity of the individual Li-Au alloy phases are not symmetrical around the point of reversal from lithiation to de-lithiation currents. This fact points to a certain hysteresis effect, i.e. a difference in reaction path between lithiation and de-lithiation of the Au thin film.

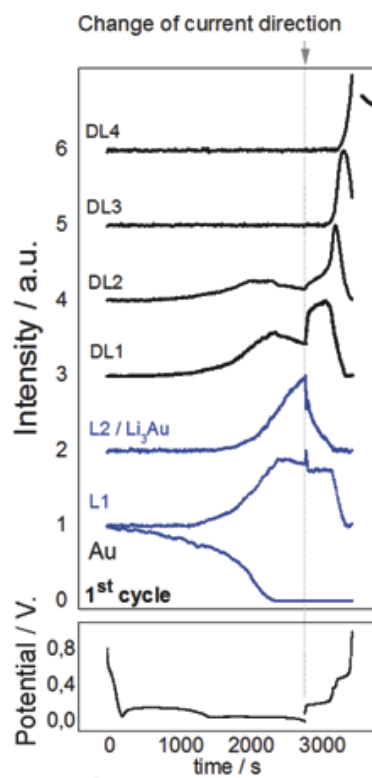

a)

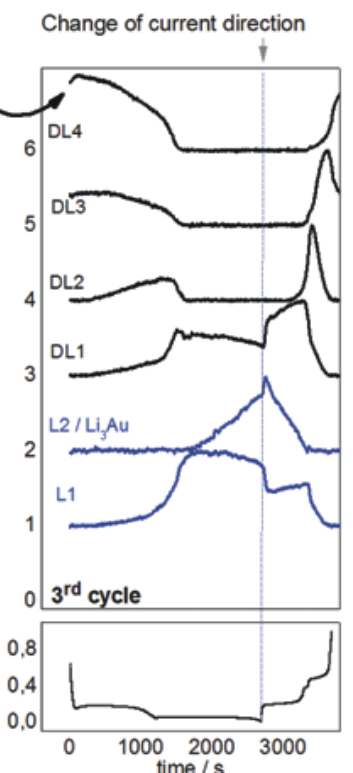

b)

Figure 3. Evolution of intensity for different Li-Au alloy phases displayed for the first (a) and the subsequent cycles (exemplary $3^{\text {rd }}$ cycle, $b$ ) with respective galvanostatic potential-time curves at bottom. 
In addition to the characteristic evolution of intensity of the alloy phases, also the signal from the pure initial $\mathrm{Au}$ is plotted in Fig. 3a. In the beginning of the lithiation cycle the intensity of Au decreases while initial alloy phases (L1 and DL1) are formed. With increasing Li content and after full consumption of the initial $\mathrm{Au}$ the initial alloy phases continue to transform into the higher lithiated L2 phase which is prevailing in the fully lithiated state (compare Fig. 3b). The simultaneous co-evolution of minority phases reveals the dynamic development of compositional inhomogeneity in the process well visible after the initial Au has been fully consumed. During the following de-lithiation, the phases L1 and DL1 are recovered, but now phase DL1 outweighs phase L1. During further de-lithiation, L1 and DL1 are transformed into phase DL2 which in turn transforms into phase DL3 during further de-lithiation. Remarkably, at the end of the cycle no Au is recovered.

Similar behavior was also observed in case of crystalline silicon anodes which are currently considered to eventually replace carbonaceous materials as LIB anodes. During the electrochemical cycles, nearly all Li-Si phases turn out to be amorphous. In these studies Si does not recover in a crystalline state after a lithiation cycle but nearly pure, amorphous $\mathrm{Si}$ is observed at the end of the de-lithiation process. As in our results on $\mathrm{Au}^{47}$ this leads to small differences between the first and subsequent galvanostatic lithiation-delithiation cycles. ${ }^{7,11,20-24,48}$ For our experiments on Au thin films a small but significant amount of Li remains in the structure giving rise to broader diffraction peaks, i.e. a structure with rather low order. A Li residue was in parallel revealed by more detailed electrochemical measurements. ${ }^{47}$ The phase DL4 appears at the end of the first cycle and is remaining when the experimental run reaches the upper potential limit of $1 \mathrm{~V}$. Consequently Li-poor amorphous Au alloy, phase DL4 and residues of DL3 are the starting material for the subsequent cycles, as shown in Figure 3.

During subsequent lithiation cycles initially the phases L1, DL1, and DL2 are reformed simultaneously. The phases DL3 and DL4 vanish quickly to the benefit of phases L1 and DL1 (at approximately $1400 \mathrm{~s}$ in Figure 3b). When phase DL2 has disappeared L2 starts growing consuming L1 and DL1. The de-lithiation halfcycle in the subsequent cycles is identical to the first cycle especially concerning the involved phase transformations. Remarkably, phases L1 and DL1 change in ratio after switching from lithiation to delithiation. As it is obvious from Fig. 4a the peak positions of phases L1, DL1, and DL2 are very similar pointing to only small structural differences between the phases. Such small energetic differences can be 
assumed based on the observed quick inter-conversion of these phases during the electrochemical cycles (Fig. 3). Then the peak positions of the DL3 phase differ remarkably from L1, DL1, and DL2, revealing clear structural differences. The sequence of the peak positions of phase L2 are similar in ratio to the Au peak positions characteristic of a face centered cubic crystal structure. Finally, the peaks showing the specific evolution of intensity of phase DL4 consist of two significantly different peak width values. This observation points to two different alloy phases showing the same evolution of intensity; one of them featuring a narrow peak width (i.e. larger grains, higher crystalline order) and the other showing broader peak width (smaller
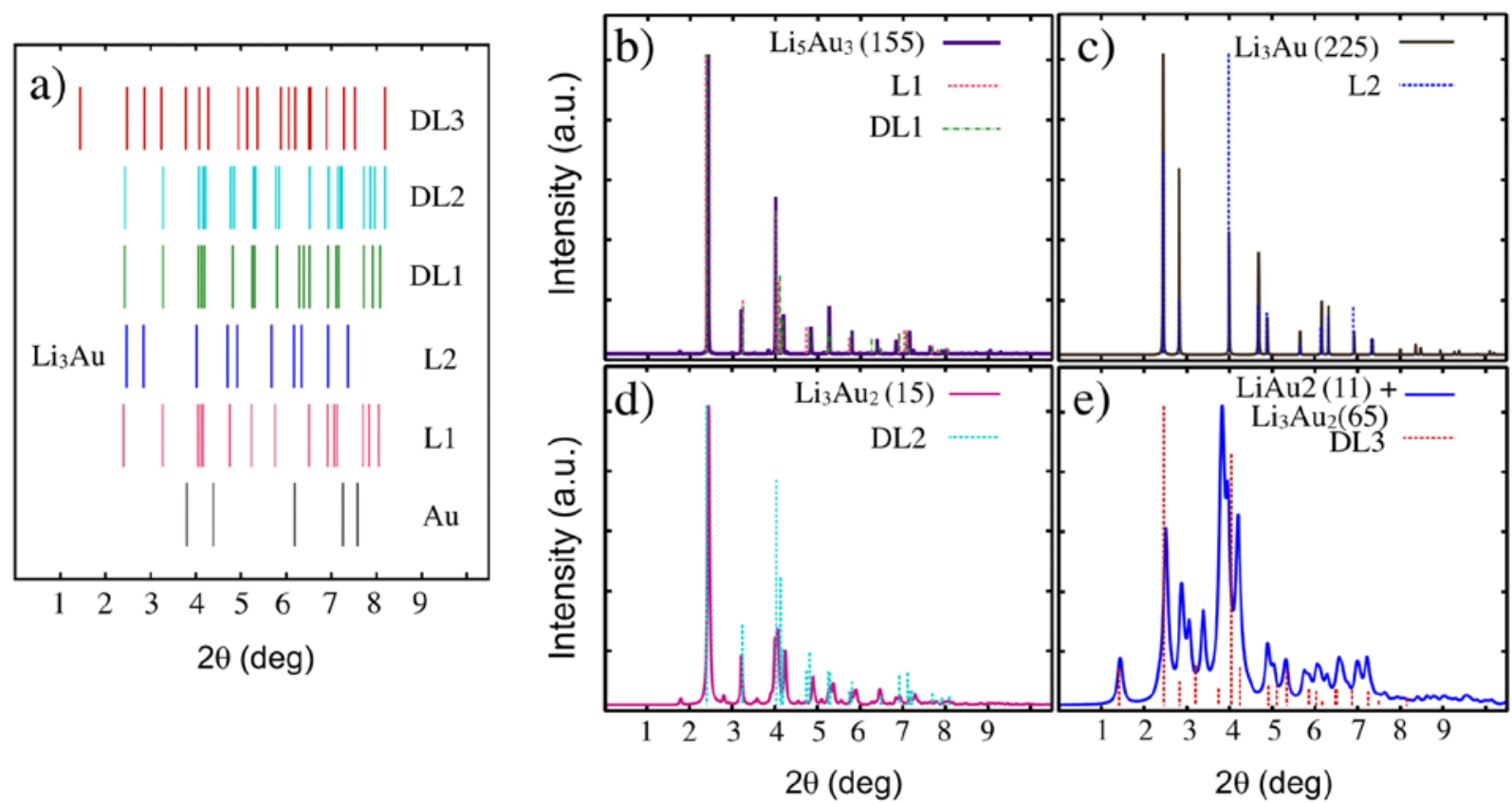

Figure 4. (Color online) a) Bragg peak positions of alloy phases as distinguished by experiment (cp. Fig. 3). L1, DL1, DL2, DL3 are metastable structural states and the associated structures are based on suggestions obtained by simulation, $\mathrm{L} 2$ is the known $\mathrm{Li}_{3} \mathrm{Au}$ phase. (b-e) Proposed structures for the observed diffraction data. $b$ ) simulated $X$-ray diffraction curve of $\mathrm{Li}_{5} \mathrm{Au}_{3}$ (space group R32) and experimental X-ray diffraction of L1 and DL1, c) simulated $X$-ray of $\mathrm{Li}_{3} \mathrm{Au}(\mathrm{Fm}-3 \mathrm{~m})$ and experimental $X$-ray diffraction of $\left.\mathrm{L} 2, \mathrm{~d}\right)$ simulated curve based on $\mathrm{Li}_{3} \mathrm{Au}_{2}(\mathrm{C12} / \mathrm{c} 1)$ and experimental $X$-ray diffraction of $\mathrm{DL2}$, e) simulated $X$-ray curve based on $\mathrm{Li}_{3} \mathrm{Au} \mathrm{u}_{5}(\mathrm{Cmmm})$ and $\mathrm{LiAu}_{2}(\mathrm{P} 121 / \mathrm{m1})$ and experimental X-ray diffraction of DL3. grains, lower crystalline order). In the following we do not further focus on this state. Figure 4a displays the positions of the successively occurring diffraction peaks ordered in groups according to the different phases L1, L2, DL1, DL2, and DL3, i.e. the separated different traces of the peak intensity evolution with time of Figure 3. The measured diffraction peaks of the initial Au relate to the expected face- 
centered cubic structure. Directly visible is the similar pattern of the second phase L2 emerging during lithiation, which can be related to the well-known cubic $\mathrm{Li}_{3} \mathrm{Au}$ phase. All other diffraction patterns cannot be related to any known, thermodynamically stable $\mathrm{Li}-\mathrm{Au}$ phase.

In order to assist to the structure determination, we performed theoretical structural search calculations. For such purpose, structures with negative formation energy (or enthalpy if pressure is present in the calculation) are found through the minima hopping method (MHM). ${ }^{41,42}$ MHM is capable of predicting stable as well as metastable crystal structures at a given pressure, from the sole knowledge of the chemical composition of the system and it has reproduced reported experimental results or found novel structures in many different systems. ${ }^{49-51}$ After the calculation, the energy per atom for all considered compositions are calculated with respect to the pristine crystals ( $\mathrm{Li}$-BCC and Au-FCC). Based on this, the so-called convex hull is constructed, which are all structures with the lowest enthalpy of formation for the specific stoichiometry but that are also more stable with respect to neighbor compositions. We should stress that one of the most important result in this calculation is not only the ground state for each composition but also a set of metastable states that can be used to understand the dynamical evolution of alloys, as it is the case in this work.
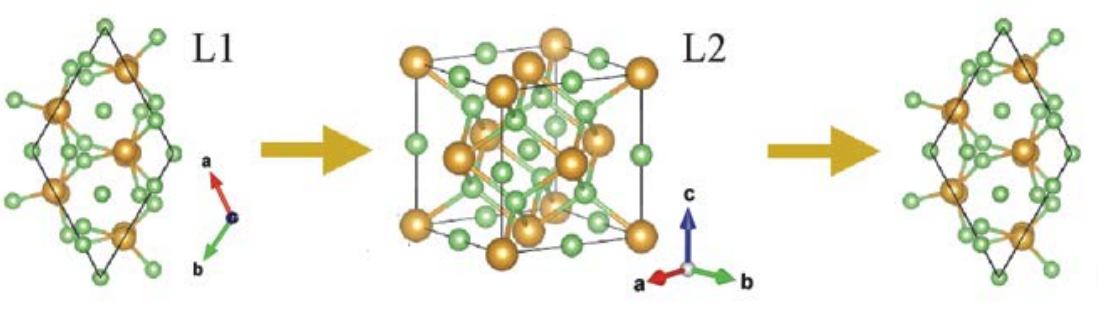

DL1

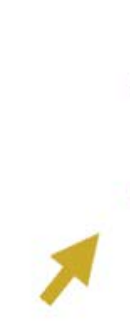

\section{DL4}
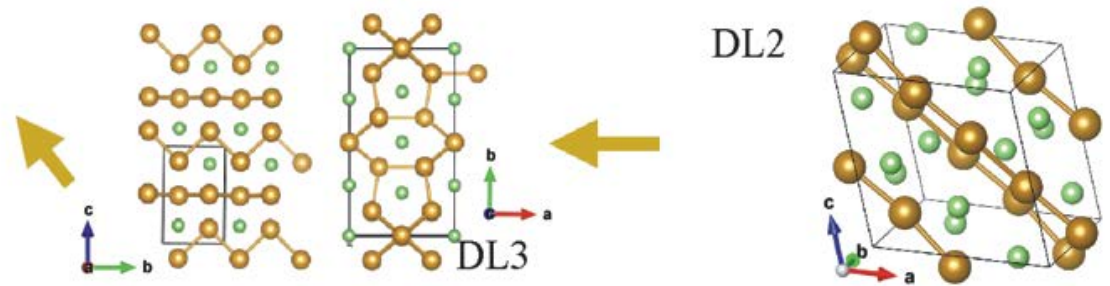

Figure 5. (Color online) Structures involved in the lithiation and de-lithiation cycles as deduced according to our experimental and theoretical work.

In Figure 4b, the X-ray diffraction pattern of L1 (pink dash lines) and DL1 (green dash lines, both structures are closely related) are contrasted to the suggested theoretical diffractogram. For this comparison, we have used the $\mathrm{Li}_{5} \mathrm{Au}_{3}$ phase (purple solid line) with space group number 155 (R32). A reasonably good agreement between this theoretical spectrum and the L1 and DL1 experimental XRD-spectra is observed. Figure 4c shows the experimental X-ray diffraction of L2 (blue dash lines) and the simulated X-ray diffraction pattern 
of the thermodynamically stable $\mathrm{Li}_{3} \mathrm{Au}$ phase (brown dash line) with space group 225 (Fm-3m). Here a very good match between experimental and the simulated pattern is noted. Earlier electrochemical measurements have already indicated that the $\mathrm{Li}_{3} \mathrm{Au}$ phase can be formed during lithiation of $\mathrm{Au}^{47}$. Figure $4 \mathrm{~d}$ then shows the simulated X-ray spectra of $\mathrm{Li}_{3} \mathrm{Au}_{2}$ with space group 15 (C12/c1). The simulated data (pink solid line) demonstrates again reasonably good match with the experimental spectra of DL2 (cyan bars). Finally, Figure 4e plots the experimental spectra of DL3 (red dash lines) and the simulated X-ray diffraction curve obtained by considering a linear combination of the theoretical diffractograms of $\mathrm{Li}_{3} \mathrm{Au}_{5}$ with space group 65 (Cmmm) and $\mathrm{LiAu}_{2}$ with space group 11 (P121/m1). To obtain this pattern, we have optimized the content of each phase with the idea of obtaining the best agreement with the obtained XRD. In particular, we have found that a combination of $90 \% \mathrm{Li}_{3} \mathrm{Au}_{5}$ with $10 \% \mathrm{LiAu}_{2}$ allow a fair agreement between the simulated and measured XRD, as shown with the blue line. From our simulations, we can conclude that the $\mathrm{Li}_{5} \mathrm{Au}_{3}(\mathrm{R} 32)$, $\mathrm{Li}_{3} \mathrm{Au}_{2}(\mathrm{C} 12 / \mathrm{c} 1), \mathrm{Li}_{3} \mathrm{Au}_{5}(\mathrm{Cmmm})$ but also $\mathrm{Li}_{3} \mathrm{Au}(\mathrm{Fm}-3 \mathrm{~m})$, are chemically meta-stable structures, and $\mathrm{LiAu}_{2}$ (P121/m1) is stable because it lies on the convex hull. A complete discussion of all obtained structures is out of scope of this report and will be published elsewhere.

In Figure 5, we recognize the atomic arrangement of the $\mathrm{Li}_{x} \mathrm{Au}_{\mathrm{y}}$ compounds proposed in the lithiationdelithiation process. In the first step of alloying (L1), we found a hexagonal structure with composition $\mathrm{Li}_{5} \mathrm{Au}_{3}$ with lattice parameters $\mathrm{a}=\mathrm{b}=7.359 \AA$ and $\mathrm{c}=8.334 \AA$. As a structural element we can distinguish chains of $\mathrm{Li}-\mathrm{Au}$, at a distance of $2.6 \AA$, and Au-Au of $2.79 \AA$. For the second appearing phase (L2) the data corresponds to the known, thermodynamically stable fcc $\mathrm{Li}_{3} \mathrm{Au}$ and a lattice parameter of $\mathrm{a}=6.282 \AA$ with a $\mathrm{Li}-\mathrm{Au}$ distance of $2.72 \AA . \mathrm{Li}_{3} \mathrm{Au}$ has been previously reported. ${ }^{23,30}$ With the reversal of the galvanostatic current direction to de-lithiaton, the first structural state which appears, is DL1. During de-lithiation state DL2, we can see from $\mathrm{Li}_{3} \mathrm{Au}_{2}(\mathrm{C} 12 / \mathrm{cm} 1)$, the formation of $\mathrm{Au}-\mathrm{Au}$ chains which are along the $<010>$ direction with Au-Au distances of 2.82 and $2.73 \AA$ and with a Li-Li distance of $2.81 \AA$. In the state DL3 we have used two different phases to explain the observed XRD diffractogram. In this case we found $\mathrm{Li}_{3} \mathrm{Au}_{5}$, (Cmmm) with five and six Au coordinated with Au-Au distances of 2.97, 2.95, and $3.06 \AA$. The other phase is $\mathrm{LiAu}_{2}(\mathrm{P} 121 / \mathrm{m})$, where we observe the formation of alternate layers of four-fold coordinated Au and Au in zig-zag chains along the c-axis. The Au-Au distance on the four-fold layer is $2.83 \AA$ while it is $2.79 \AA$ in the zig-zag chains.

In order to understand the evolution for the first and subsequent cycles of the Li-Au system, it is worth to have a closer look into the rearrangement of the Au atoms of the suggested structures. Figure 6a displays the Au atoms of the L1, L2, DL1, DL2, and DL3 phases in the (001), (111), (001), (111), and (001) + (001) planes, respectively. In the L1 phase we observe along the (001) plane the formation of a hexagonal lattice, where the Au-Au distances are 4.02 and $4.37 \AA$. Then in the L2 phase we again have hexagon formation on 
the (111) planes with Au-Au distances of $4.44 \AA$. In DL1 we observe the same basic Au structure as in L1. In the DL2 phase we can observe the formation of a distorted structure, with two different environments for two of the $\mathrm{Au}$ atoms, each fourfold coordinated as in $\mathrm{Li}_{3} \mathrm{Au}_{2}$ with $\mathrm{Au}-\mathrm{Au}$ distances of 3.92, 2.82, 2.73, and 4.11 $\AA$ and 4.11, 2.73, 4.57 and $2.82 \AA$, respectively. In the DL3 phase, the theoretically predicted crystal structures that are used to explain the experimental XRD have very similar Au arrangements, where fourfold coordinatated $\mathrm{Au}$ atoms are noted in Figure 6a, along the 001 plane. For this last phase, the Au-Au distances in $\mathrm{LiAu}_{2}$ are 2.82 and $2.83 \AA$ while in $\mathrm{Li}_{3} \mathrm{Au}_{5}$ we have in-equivalent Au-Au distances of 3.24, 2.97, 3.06 and $2.97 \AA$. A summary of the crystallographic data is given in Table 1. Moreover, the Wyckoff positions are detailed in supplementary information (Table S1). In addition supplementary information presents an approximated average potential which can be predicted based on simple assumptions from the theoretical structure calculations (Fig. S1) and fairly reproduces the observed potentials.
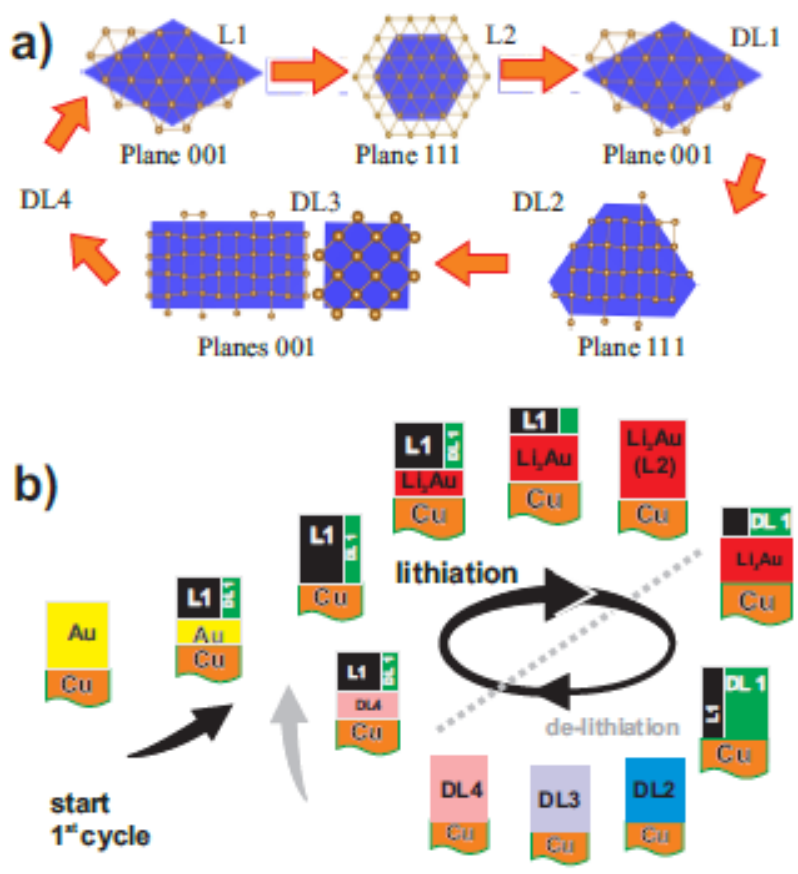

Figure 6. (Color online) Structural elements and phase evolution of the Gold-Lithium alloy phases during the electrochemical cycles. a) Details of the structural evolution showing close-packed Au planes based on proposed structures. b) Phase Evolution for the Au-Li system during our galvanostatic experiments.

The presented structural sequence allows for smooth transformations between the different stages and the related unit cells. The results of our X-ray study here are in line with electrochemical characterization reported earlier ${ }^{28,29}$. During our in-situ high-energy X-ray study, but also during our earlier electrochemical tests $^{47}$, the used initial Au thin-film substrates and the chosen galvanostatic anode lithiation (charging) and de-lithiation (discharging) protocols result in the presented, well reproducible sequence of structural states 
(Figure 6b). Some conflicting results on the alloy structures formed during reaction of Li and Au reported in the literature $\mathrm{e}^{30-32}$ can in part be explained by different experimental conditions, such as potentiostatic instead of galvanostatic modes. Also different sample geometries, as for example nanoparticles versus thin films, influence the alloy formation due to different possibilities for stress relaxation ${ }^{32}$.

Table 1.Space group, lattice parameters and angles for the unit cells of the proposed structures.

\begin{tabular}{|c|c|c|c|c|c|c|c|}
\hline Phase & $\begin{array}{l}\text { Space } \\
\text { group }\end{array}$ & $\mathrm{a}$ & $\mathrm{b}$ & C & $\alpha$ & $\gamma$ & $\beta$ \\
\hline $\begin{array}{c}\mathrm{Li}_{5} \mathrm{Au}_{3} \\
(\mathrm{~L} 1)\end{array}$ & R32, 155 & $\begin{array}{c}7 . \\
35 \\
9\end{array}$ & 7.359 & $\begin{array}{c}8 . \\
33 \\
4\end{array}$ & $\begin{array}{l}9 \\
0\end{array}$ & 90 & 1 \\
\hline $\mathrm{Li}_{3} \mathrm{Au}(\mathrm{L} 2)$ & $\begin{array}{c}\mathrm{F} m-3 m \\
225\end{array}$ & $\begin{array}{c}6 . \\
28 \\
2\end{array}$ & 6.282 & $\begin{array}{c}6 . \\
28 \\
2\end{array}$ & $\begin{array}{l}9 \\
0\end{array}$ & 90 & 9 \\
\hline $\begin{array}{c}\mathrm{Li}_{3} \mathrm{Au}_{2} \\
(\mathrm{DL} 2)\end{array}$ & $\mathrm{C} 12 / \mathrm{c} 1,15$ & $\begin{array}{c}7 . \\
02 \\
1\end{array}$ & 7.229 & $\begin{array}{c}6 . \\
84 \\
8\end{array}$ & 0 & $\begin{array}{l}10 \\
5 . \\
4\end{array}$ & 9 \\
\hline $\begin{array}{c}\mathrm{Li}_{5} \mathrm{Au}_{3} \\
(\mathrm{DL} 2)\end{array}$ & P-62m,189 & $\begin{array}{l}7 . \\
20\end{array}$ & 7.20 & $\begin{array}{c}2 . \\
85 \\
6\end{array}$ & 0 & 90 & 1 \\
\hline $\begin{array}{c}\mathrm{Li}_{3} \mathrm{Au}_{5} \\
(\mathrm{DL} 3)\end{array}$ & $\mathrm{Cmmm,65}$ & $\begin{array}{c}7 . \\
05 \\
6\end{array}$ & $\begin{array}{c}12.36 \\
8\end{array}$ & $\begin{array}{c}2 . \\
90 \\
2\end{array}$ & $\begin{array}{l}9 \\
0\end{array}$ & 90 & 0 \\
\hline $\begin{array}{l}\mathrm{LiAu}_{2} \\
(\mathrm{DL3})\end{array}$ & $\begin{array}{c}\mathrm{P} 121 / m 1, \\
11\end{array}$ & $\begin{array}{c}3 . \\
96 \\
1\end{array}$ & 4.018 & $\begin{array}{c}6 . \\
51 \\
6\end{array}$ & $\begin{array}{l}9 \\
0\end{array}$ & $\begin{array}{c}10 \\
6 . \\
8\end{array}$ & 0 \\
\hline
\end{tabular}

With our experimental conditions, for example, we do not observe the crystalline low-Li alloy $\alpha$-AuLi phase which was occasionally reported in the literature ${ }^{30,32}$. The fcc structure of $\alpha$-AuLi is formed directly from 
structure. Moreover, the re-formation of crystalline $\mathrm{Au}$ at the end of the lithiation-delithiation cycle was reported $^{30}$. Earlier work was typically performed at lower X-ray energy compared to our high-energy diffraction study and the reported reciprocal range was correspondingly more limited and thus more difficult to associate structures. Some of the reported peaks and their behavior are in line with our phases L1, L2, or DL1 (for example the recent work by Misra et al. ${ }^{24}$ ), but also additional unknown diffraction signal was reported. $^{30,} 32$ In part, earlier results were obtained by ex-situ XRD measurements, but especially for structures involving Li with their even at room-temperature high diffusion rate, the potential-dependent alloy phase structures show a limited stability which underlines the importance and necessity of in-situ experiments.

Our reproducible patterns show clearly that in the first step of lithiation, the highly crystalline Au film completely transformed into polycrystalline or nanocrystalline alloy phases. Distinct diffraction peaks of $\mathrm{Au}$ get replaced by diffraction rings of uniform azimuthal intensity. Subsequently, the upcoming phase L2 is again characterized by distinct peaks indicating once again high crystalline (out of plane) order with in-plane texture. The high degree of texture, i.e. preferred orientations, can be directly inferred from the visibly distinct diffraction peaks related to phase $\mathrm{L} 2$, which also shows that the reciprocal plane is perpendicular to the surface. A more detailed indexing of the diffraction peaks assigned to L2 reveals, that a limited number of $\mathrm{Li}_{3} \mathrm{Au}$ crystallites is present in the sample sharing a (220) orientation parallel to the $\mathrm{Cu}(001)$ surface. The rotation of the individual crystallites around the surface normal is, however, random. From this fact it can be concluded, that phase L2 grows starting from the Cu-substrate/thin-Au-film interface, i.e. using the $\mathrm{Cu}$ surface as a template for nucleating $\mathrm{Li}_{3} \mathrm{Au}$ crystallites with a preferred parallel orientation. The limited number of crystallites in the pathway of the beam implicates a significantly larger crystallite-size compared to the other Li alloy phases. During de-lithiation the textured crystalline phase L2 transforms back into powder-like phases. The similarity of the diffraction patterns of phase L1, DL1, and DL2 indicates a high structural similarity. Our earlier electrochemical characterization of the lithiation-delithiation behavior substantiates the high energetic similarity of these three phases ${ }^{47}$. The further de-lithiation to phase DL3 is associated with larger changes in the crystal structure as indicated by the considerable change of the diffraction pattern.

\section{Conclusion}

We performed ambient-temperature in-situ high-energy X-ray diffraction experiments (XRD) as well as theoretical structural search calculations based on the minima hopping method (MHM) on the electrochemical alloying of gold with lithium. XRD experiments showed that six different alloy phases are involved during the alloying and dealloying cycles. With exception of $\mathrm{Li}_{3} \mathrm{Au}$ all observed alloy phases are 
metastable, so far unknown structures. According to our calculations $\mathrm{LiAu}_{2}$ was predicted as a stable structure lying on the convex hull. By performing MHM simulations we could propose detailed unit cells for the involved phases. The observed crystalline alloy phases could thus be assigned to thermodynamically stable $\mathrm{Li}_{3} \mathrm{Au}$ and $\mathrm{LiAu}_{2}$ and metastable $\mathrm{Li}_{5} \mathrm{Au}_{3}, \mathrm{Li}_{3} \mathrm{Au}_{2}$, and $\mathrm{Li}_{3} \mathrm{Au}_{5}$ phases. The observed metastable phases did transform when at open circuit potential demonstrating the need to perform in-situ diffraction.

\section{Acknowledgment}

Portions of this research were carried out at beamline P07 of the liht source PETRA III at DESY, a member of the Helmholtz Association (HGF). This work was in part supported by the German Federal Ministry for Education and Research (BMBF) in the framework of the Kompetenzverbund Nord project. The authors used the Extreme Science and Engineering Discovery Environment (XSEDE), which is supported by USA National Science Foundation grant number OCI-1053575. Additionally, the authors acknowledge the support from the Texas Advanced Computer Center (TACC) and Super Computing System (Mountaineer) at WVU, which are funded in part by the National Science Foundation EPSCoR Research Infrastructure Improvement Cooperative Agreement 1003907, the state of West Virginia (WVEPSCoR via the Higher Education Policy Commission) and WVU. A.H.R. and I.V.-J. acknowledge the support of NSF under project 1434897 and the Donors of the American Chemical Society Petroleum Research Fund for partial support of this research under contract 54075-ND10.

\section{References}

[1] Tarascon, J. M., Armand, M. Issues and Challenges Facing Rechargeable Lithium Batteries. Nature 2001, 414, 359-367.

[2] Goodenough, J. B., Kim, Y. Challenges for Rechargeable Li Batteries. Chem. Mater., 2010, 22, 587-603.

[3] Marom, R., Amalraj, S.F., Leifer, N., Jacob, D., Aurbach, D. A Review of Advanced and Practical

Lithium Battery Materials. J. Mater. Chem. 2011, 21, 9938-9954.

[4] Wagner R., Preschitschek, N., Passerini, S., Leker, J., Winter, M. Current Research Trends and Prospects Among the Various Materials and Designs Used in Lithium-Based Batteries. J. Appl. Electrochem, 2013, 43, 481-496.

[5] Deng, D. Li-Ion Batteries: Basics, Progress, and Challenges. Energy Sci Eng. 2015, 3, 385-418.

[6] McDowell, M.T.; Lee, S. W.; Nix W. D.; Cui, Y. Understanding the Lithiation of Silicon and Other Alloying Anodes for Lithium-Ion Batteries. Adv. Mater. 2013, 25, 4966-4985. 
[7] Chan, C.K.; Peng, H.; Liu, G.; McIlwrath, K.; Zhang, X.F.; Huggins, R.A.; Cui, Y. High-Performance Lithium Battery Anodes Using Silicon Nanowires. Nat. Nanotech. 2008, 3, 31-35.

[8] Obrovac, M.N.; Chevrier, V.L. Alloy Negative Electrodes for Li-Ion Batteries. Chem. Rev. 2014, 114, 11444-11502.

[9] Goriparti, S.; Miele, E., De Angelis, F.; Di Fabrizio, E.; Proietti Zaccaria, R.; Capiglia, C. Review on Recent Progress of Nanostructured Anode Materials for Li-ion Batteries. J. Power Sources 2014, 257, 421443.

[10] Rubloff, G.W., Lee, S.B. New Science at the Meso Frontier: Dense Nanostructure Architectures for Electrical Energy Storage. Curr. Op. Solid. State Mater. Sci. 2015, 19, 227-234.

[11] Graetz, J.; Ahn, C. C.; Yazami, R.; Fultz B. Highly Reversible Lithium Storage in Nanostructured Silicon. Electrochem. Solid St. Lett. 2003, 6 A194-A197.

[12] Kalyanasundaram K.; Grätzel, M. Themed Issue: Nanomaterials for Energy Conversion and Storage. $J$. Mater. Chem. 2012, 22, 24190-24194.

[13] Borissov, D.; Pareek, A.; Renner, F. U.; Rohwerder, M. Electrodeposition of Zn and Au-Zn Alloys at Low Temperature in an Ionic Liquid. Phys. Chem. Chem. Phys. 2010. 12 2059-2062.

[14] Oezaslan, M., Heggen, M., Strasser, P. Size-Dependent Morphology of Dealloyed Bimetallic Catalysts: Linking the Nano to the Macro scale.. J. Am. Chem. Soc. 2011, 134, 514-524.

[15] Nishio, K.; Yuda, K.; Masuda, H. Fabrication of Nanoporous Pt by Electrochemical Alloying and Dealloying with Li. ECS Electrochem. Lett. 2013, 2, C43-C45.

[16] Song, T.; Yan, M.; Gao, Y.; Atrens, A.; Qian, M. Concurrence of De-Alloying and Re-Alloying in a Ternary Al67Cu18Sn15 Alloy and the Fabrication of 3D Nanoporous Cu-Sn Composite Structures. RSC Advances 2015, 5, 9574-9580.

[17] Ankah, G.N.; Pareek A.; Cherevko S.; Zegenhagen J.; Renner, F.U. Hierarchical Nanoporous Films Obtained by Surface Cracking on $\mathrm{Cu}-\mathrm{Au}$ and Ethanethiol on $\mathrm{Au}(001)$. Electrochim. Acta 2014, 140, 352358.

[18] Renner F.U.; Ankah G.N.; Bashir A.; Ma D.; Biedermann P.U.; Shrestha B.R.; Nellessen M.;

Khorashadizadeh A.; Losada-Pérez P.; Duarte M.J.; Raabe D.; Valtiner M. Star-Shaped Crystallographic Cracking of Localized Nanoporous Defects. Adv Mater. 2015, 27, 4877-4882.

[19] Qi, Z.; Weissmüller J. ACS Nano 2013, 7, 5948.

[20] Obrovac, M. N.; Krause L. J. Reversible Cycling of Crystalline Silicon Powder. J. Electrochem. Soc. 2007, 154, A103-A108.

[21] Hatchard, T. D.; Dahn J. R. In Situ XRD and Electrochemical Study of the Reaction of Lithium with Amorphous Silicon. J. Electrochem. Soc. 2004, 151, A838-A842.

[22] Li, J.; Dahn, J.R. An In Situ X-ray Diffraction Study of the Reaction of Li with Crystalline Si. J. 
Electrochem. Soc. 2007, 154, A156-A161.

[23] Jung, H.; Allan, P. K.; Hu, Y.-Y.; Borkiewicz, J. O.; Wang, X.-L.; Han, W.-Q.; Du, L.-S.; Pickard, C. J.; Chupas, P. J.; Chapman, K. W.; Morris, A. J.; Grey, C. P. Elucidation of the Local and Long-Range Structural Changes that Occur in Germanium Anodes in Lithium-Ion Batteries. Chem. Mater., 2015, 27, 1031-1041.

[24] Misra, S.; Liu, N.; Nelson, J.; Hong, S.S.; Cui, Y.; Toney, M. F. In situ X-ray Diffraction Studies of (De)Lithiation Mechanism in Silicon Nanowire Anodes. ACS Nano 6 (2012) 5465-5473.

[25] Zeng, Z.Y.; Liang, W.I.; Liao, H.G.; Xin, H.L.L.; Chu Y.H.; Zheng, H.M. Visualization of ElectrodeElectrolyte Interfaces in LiPF6/EC/DEC Electrolyte for Lithium Ion Batteries Via In-Situ TEM. Nano Lett., 2014, 14, 1745-1750.

[26] Zeng, Z.Y.; Liang, W.I.; Chu, Y.-H.; Zheng, H.M. In situ TEM Study of the Li-Au Reaction in an Electrochemical Liquid Cell. Faraday Discuss. 2014, 176, 95-107.

[27] Chou, C.-Y.; Seo, J.-H.; Tsai, Y.-H.; Ahn, J.-P.; Paek, E.; Cho, M.-H.; Choi, I.-S.; Hwang, G. S. Anomalous Stagewise Lithiation of Gold-Coated Silicon Nanowires: A Combined In Situ Characterization and First-Principles Study. ACS Appl. Mater. Interfaces 2015, 7, 16976-16983.

[28] Thakur, M.; Isaacson,M.; Sinsabaugh, S.L.; Wong, M.S.; Biswal, S.L. Gold-Coated Porous Silicon Films as Anodes for Lithium Ion Batteries. J. Power Sources 2012, 205, 426-432.

[29] Yu, Y.; Gu, L.; Lang, X.; Zhu, C.; Fujita, T.; Chen, M.W.; Maier, J. Li Storage in 3D Nanoporous AuSupported Nanocrystalline Tin. Adv. Mater. 2011, 23 2443-2447.

[30] Taillades, G.; Nejelloun, N.; Sarradin, J.; Ribes, M. Metal-Based very Thin Film Anodes for Lithium Ion Microbatteries. Solid State Ionics 2002, 152-153, 119-124.

[31] Yuan, L.; Liu, H.K.; Maaroof, A.; Konstantinov, K.; Liu, J.; Cortie, M.J. Mesoporous Gold as Anode Material for Lithium-Ion Cells. New Mat. Elect. Syst. 2007, 10, 95-99.

[32] Renner, F.U.; Kageyama, H.; Siroma, Z.; Shikano, M.; Schröder, S.; Gründer, Y.; Sakata, O. Gold Model Anodes for Li-Ion Batteries: Single Crystalline Systems Studied by In Situ X-ray Diffraction. Electrochim. Acta 2008, 53, 6064-6069.

[33] Gustafson, J.; Shipilin,M.; Zhang,C.; Stierle, A.; Hejral, U.; Ruett, U.; Gutowski, O.; Carlsson, P.-A.; Skoglundh, M.; Lundgren, E. High-Energy Surface X-ray Diffraction for Fast Surface Structure Determination. Science 2014, 343, 758-761.

[34] Mezger, M.; Schröder, H.; Reichert, H.; Schramm, S.; Okasinski, J.S.; Schöder, S.; Honkimäki, V.; Deutsch, M.; Ocko, B.M.; Ralston, J.; Rohwerder, M.; Stratmann, M.; Dosch, H. Molecular Layering of Fluorinated Ionic Liquids at a Charged Sapphire (0001) Surface. Science 2008, 322, 424-428. 
[35] Zhou, Q.; Boyle, P.D.; Malpezzi, L.; Mele, A.; Shin, J.-H., Passerini, S.; Henderson, W.A. Phase Behavior of Ionic Liquid-LiX Mixtures: Pyrrolidinium Cations and TFSI- Anions - Linking Structure to Transport Properties. Chem. Mat. 2011, 23, 4331-4337.

[36] Xu, K. Electrolytes and Interphases in Li-Ion Batteries and Beyond. Chem. Rev. 2014, 114, 1150311618.

[37] Henderson, W.A.; Passerini, S. Phase Behavior of Ionic Liquid-LiX Mixtures: Pyrrolidinium Cations and TFSI- Anions. Chem. Mater. 2004, 16, 2881-2885.

[38] Renner, F.U.; Gründer, Y.; Zegenhagen, J. Portable Chamber for the Study of UHV Prepared Electrochemical Interfaces by Hard X-ray Diffraction. Rev. Sci. Instr. 2007, 78, 33903(1-8).

[39] Bach, P.; Seemayer, A.; Rütt, U.; Gutowski, O.; Renner, F.U. In-Situ XRD Investigation of Insertion and Extraction Mechanisms of Lithium in Nanoscaled Al Thin Films. ECS Trans. 2013, 53, 29-39.

[40] Jennings, G. QXRD Readout Software for Flat Panel X-ray Detectors. Software and description is available at http://qxrd.sourceforge.net

[41] Goedecker S. J. Minima Hopping: an Efficient Search Method for the Global Minimum of the Potential Energy Surface of Complex Molecular Systems. Chem. Phys. 2004, 120, 9911-9917.

[42] Amsler, M.; Goedecker, S. Crystal Structure Prediction Using the Minima Hopping Method. J. Chem. Phys. 2010, 133, 224104 (1-8).

[43] Kresse, G.; Furthmüller, J. Efficient Iterative Schemes for Ab Initio Total-Energy Calculations Using a Plane-Wave Basis Set. Phys. Rev. B 1996, 54, 11169-11186.

[44] Kresse, G.; Joubert, D. From Ultrasoft Pseudopotentials to the Projector Augmented-Wave Method. Phys. Rev. B 1999, 59, 1758-1775.

[45] Blöchl, P. E. Projector Augmented-Wave Method. Phys. Rev. B 1994, 50, 17953-17979

[46] Perdew, J.P.; Burke, K.; Ernzerhof, M. Generalized Gradient Approximation Made Simple. Phys. Rev. Lett. 1996, 77, 3865-3868.

[47] Bach, P.; Stratmann, M.; Valencia-Jaime, I.; Romero, A.H.; Renner, F.U. Lithiation and Delithiation Mechanisms of Gold Thin Film Model Anodes for Lithium Ion Batteries: Electrochemical Characterization. Electrochim. Acta 2015, 164, 81-89.

[48] A. Netz, R. A. Huggins, W. Weppner, The formation and properties of amorphous silicon as negative electrode reactant in lithium systems, J. Power Sources 119-121 (2003) 95.

[49] Sarmiento-Pérez, R.; Cerqueira, T. F. T.; Valencia-Jaime, I.; Amsler, M.; Goedecker, S.; Romero, A. H.; Botti, S.; Marques, M.A.L. Novel Phases of Lithium-Aluminum Binaries from First-Principles Structural Search. J. Chem. Phys. 2015, 142, 024710 (1-6). 
[50] Sarmiento-Pérez, R.; Cerqueira, T.F.T.; Valencia-Jaime, I.; Amsler, M.; Goedecker, S.; Botti, S.; Marques, M.A.L.; Romero, A.H. Sodium-Gold Binaries: Novel Structures for Ionic Compounds from an Ab Initio Structural Search. New J. Phys. 2013, 15, 115007 (1-9).

[51] Amsler, M.; Flores-Livas, J. A.; Huan, T. D.; Botti, S.; Marques, M. A. L.; Goedecker, S. Novel Structural Motifs in Low Energy Phases of LiAlH4. Phys. Rev. Lett. 2012, 108, 205505.

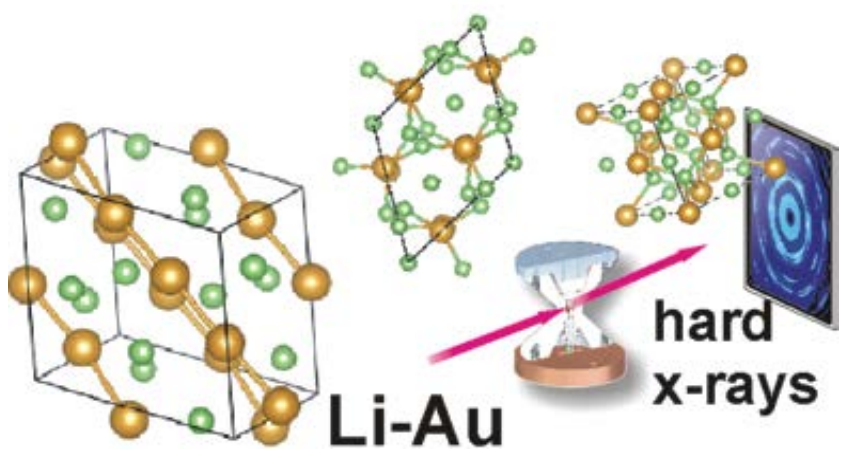




\title{
Supplementary Information
}

for

\section{Electrochemical Lithiation Cycles of Gold Anodes Observed by In- Situ High-Energy X-ray Diffraction}

\author{
Philipp Bach ${ }^{1,2,31}$, Irais Valencia-Jaime ${ }^{4,51}$, Uta Rütt ${ }^{6}$, Olof Gutowski ${ }^{6}$, \\ Aldo H. Romero ${ }^{5}$, Frank U. Renner ${ }^{* 1,2,7}$ \\ ${ }^{1}$ Institute for Materials Research (IMO), Hasselt University, Wetenschapspark 1, 3590 Diepenbeek, Belgium \\ ${ }^{2}$ Department of Interface Chemistry and Surface Engineering, Max-Planck Institut für Eisenforschung, Max-Planck- \\ Straße 1, 40237 Düsseldorf, Germany \\ ${ }^{3}$ Center for Electrochemical Sciences - CES, Ruhr-Universität Bochum, Universitätsstraße 150, 44780 Bochum, \\ Germany \\ ${ }^{4}$ Centro de Investigacion y Estudios Avanzados del IPN, MX-76230, Querétaro, México \\ ${ }^{5}$ Department of Physics and Astronomy, West Virginia University, Morgantown, WV-26506-6315, USA \\ ${ }^{6}$ German Electron Synchrotron (DESY), Notkestraße 85, 22607 Hamburg, Germany \\ ${ }^{7}$ IMEC vcs IMOMEC, Wetenschapspark 1, 3590 Diepenbeek, Belgium \\ ${ }^{*}{ }^{\dagger}$ These authors contributed equally; \\ Corresponding author: Email address: frank.renner@uhasselt.be (F.U.R.)
}


Table S1. Space group, and Wyckoff positions for the unit cells of the proposed structures.

\begin{tabular}{|c|c|c|c|c|c|}
\hline Phase & & $\begin{array}{l}\text { Wyckoff } \\
\text { site }\end{array}$ & $\mathrm{x}$ & $\mathrm{y}$ & $\mathrm{z}$ \\
\hline \multirow{3}{*}{$\begin{array}{l}\mathrm{Li}_{5} \mathrm{Au}_{3}, 155 \\
(\mathrm{~L} 1)\end{array}$} & Au1 & $9 e$ & 0.68469 & 0.00000 & 0.50000 \\
\hline & Li1 & $6 c$ & 0.00000 & 0.00000 & 0.30944 \\
\hline & Li2 & $9 d$ & 0.26223 & 0.00000 & 0.00000 \\
\hline \multirow{3}{*}{$\begin{array}{l}\mathrm{Li}_{3} \mathrm{Au}, 225 \\
(\mathrm{~L} 2)\end{array}$} & Au1 & $4 a$ & 0.00000 & 0.00000 & 0.00000 \\
\hline & Li1 & $4 \mathrm{~b}$ & 0.50000 & 0.50000 & 0.50000 \\
\hline & Li2 & $8 c$ & 0.25000 & 0.25000 & 0.25000 \\
\hline \multirow{3}{*}{$\begin{array}{l}\mathrm{Li}_{3} \mathrm{Au}_{2}, 15 \\
(\mathrm{DL} 2)\end{array}$} & Au1 & $8 f$ & 0.86897 & 0.27606 & 0.36603 \\
\hline & Li1 & $8 f$ & 0.69230 & 0.43662 & -0.01671 \\
\hline & Li2 & $4 \mathrm{e}$ & 0.00000 & 0.63232 & 0.25000 \\
\hline \multirow{4}{*}{$\begin{array}{l}\mathrm{Li}_{3} \mathrm{Au}_{5}, 65 \\
(\mathrm{DL} 3)\end{array}$} & Au1 & $8 q$ & 0.71711 & 0.36873 & 0.50000 \\
\hline & Au2 & $2 b$ & 0.50000 & 0.00000 & 0.00000 \\
\hline & Li1 & $4 i$ & 0.00000 & 0.26969 & 0.00000 \\
\hline & $\mathrm{Li} 2$ & $2 a$ & 0.00000 & 0.00000 & 0.00000 \\
\hline \multirow{3}{*}{$\begin{array}{l}\mathrm{LiAu}_{2}, 11 \\
\text { (DL3) }\end{array}$} & Au1 & $2 e$ & -0.07985 & 0.25000 & 0.84498 \\
\hline & Au2 & 2e & 0.24724 & 0.25000 & 0.49290 \\
\hline & Li1 & $2 e$ & 0.59344 & 0.25000 & 0.18175 \\
\hline
\end{tabular}




\section{Voltage curve}

\section{Experimental details}

In order to relate the experimental galvanostatic lithiation and delithiation behavior with the theoretical results, we calculated the electrical potential profile. Similar to an approach for $\mathrm{Si}$ alloys [Zhang2013b,Courtney1998] we assume that the average cell potential can be predicted under the assumption that the transformation from reactants $\left(\mathrm{Li}_{\mathrm{x}} \mathrm{Au}\right)$ to product $\left(\mathrm{Li}_{\mathrm{y}} \mathrm{Au}\right)$ is a two phase reaction.

$$
L i_{x}+(x / y) A u \rightarrow L i_{y} A u
$$

Where the average voltage is defined as

$$
\text { Voltage }=-\left\{\frac{G_{L i_{y} A u}-G_{L i_{x} A u}}{y-x}-G\left(L i_{\text {metal }}\right)\right\}
$$

With the Gibbs energy $G$ defined as

$$
G=E+p V-T S
$$

Where $E$ is energy, $V$ is volume, $\mathrm{p}$ is pressure, $T$ is temperature and $S$ is entropy. Here, the contribution $p V$ to $G$ is of the order of $10 \mu \mathrm{eV}$ per $\mathrm{Li}$ at atmospheric pressure, and the contribution of TS is of the order of 10 $m e V$, i.e. both are of comparably small value. Therefore, the considered approximated average potential in our calculations is given by:

$$
\text { Voltage }=E(L i)-\frac{E\left(L i_{y} A u\right)-E\left(L i_{x} A u\right)}{y-x}
$$

Where the total energies have been calculated by means of electronic structure calculations within DFT.

In Figure S1 we show a comparison between the experiment and simulated lithiation/delithiation process. The obtained theoretical results are always larger than the obtained experimental values. This can be due mainly for two reasons: (i) the theoretical equations used to calculate the voltage assume equilibrium conditions and that the crystal phases are single or very well mixed. It is not clear that those conditions are present in the experiment, in particular the surfaces may be different from deeper parts of the alloy. For the second main possible reason, (ii) our theoretical calculations assumed that the crystals extend to infinity and there are no grain boundaries, defects or vacancies that could affect the ionic diffusion properties. In contrast to other lithium batteries alloys such as Li-Si [Wen1981, Okamoto2009, Nesper1987] and Li-Sn [Courtney1998] where the transition of phases in the electrochemical process corresponds to the equilibrium phases in the phase diagram, in our work some of the phases that explain the in-situ XRD data do not 
correspond to equilibrium states and they do not lie on the convex hull. In any case, we see clearly that our predicted voltage follows the main trend and it predicts major transitions in voltage where the experiment also observes changes.

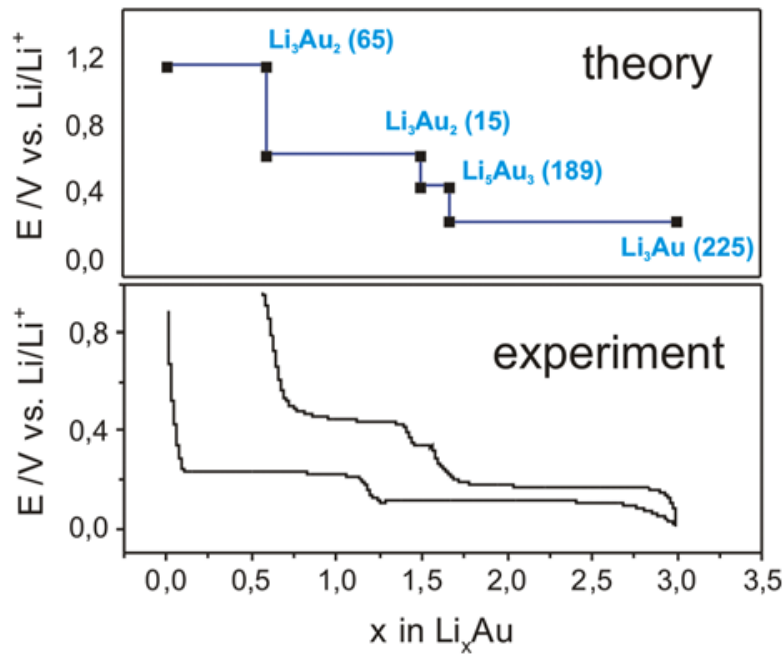

Figure $\mathbf{S 1}$ Theoretical approximation and experimental potential behavior.

[Zhang2013b] Q. Zhang, Y. Cui, and E. Wang, Modelling and Simulation in Materials Science and Engineering 21, 074001 (2013).

[Courtney1998] I. A. Courtney, J. S. Tse, O. Mao, J. Hafner, and J. R. Dahn, Phys. Rev. B 58, 15583 (1998)

[Wen1987] C. J. Wen and R. A. Huggins, Journal of Solid State Chemistry 37, 271 (1981).

[Okamoto] H. Okamoto, Journal of Phase Equilibria and Diffusion 30, 118 (2009).

[Nesper ] R. Nesper and H. G. von Schnering, Journal of Solid State Chemistry 70, 48 (1987) 